\title{
Automation of Image Processing for Nano-beam Diffraction Measurements
}

\author{
Darren Homeniuk ${ }^{1}$, Francisco Paraguay Delgado ${ }^{2}$, Marek Malac ${ }^{1,3}$ and Misa Hayashida ${ }^{1}$ \\ 1. Nanotechnology Research Center, 11421 Saskatchewan Drive, T6G 2M9, Edmonton, Alberta, Canada \\ 2. Centro de Investigación en Materiales Avanzados S C Laboratorio Nacional de Nanotechnología, \\ Miguel de Cervantes 120, C P, 31136 Chihuahua, México \\ 3. Department of Physics, University of Alberta, T6G 2E1, Edmonton, Alberta, Canada
}

Parallel nanometer-beam diffraction (NBD) in a scanning transmission electron microscope (STEM) produces vast quantities of potentially useful data. A MATLAB toolbox was developed for automated data collection and analysis and is applied to a sample containing nanoparticles (NPs). The NPs are automatically located in three dimensions (3D) by STEM electron tomography, are automatically tracked between tilts, and then NBD patterns are collected at several sample tilts allowing for the identification of the NP structure.

A typical parallel NBD data set contains sample images and diffraction patterns from selected areas, such as individual NPs. The data can be used to investigate the NP morphology and local crystal structure [1]. Typically the analysis is performed manually. An alternative is to automate the processes of STEM image collection, NP detection, diffraction pattern collection and analysis, as well as unit cell calculations in MATLAB, which greatly increases the data collection speed and reduces opportunities for human error. The experiments were performed on a Hitachi HF-3300 TEM / STEM with cold field emission gun and MAESTRO central computer control system operated at $300 \mathrm{kV}$ [2][3].

The first step in NBD is locating the NPs in STEM images. Example images are shown in Fig 1, where some of the NPs are located by the software with green crosses. Locating the center of the particles is crucial so that good diffraction patterns are collected when the nanometer sized parallel beam is placed at the detected NP location. To detect the NPs accurately, a morphological background subtraction is implemented to even out the variations in the background of the image. A low pass filter removes any high-noise areas in the background. A user-defined threshold extracts the particles from the background of the image, generating a binary image. Groups of contiguous particle pixels are grouped together, centroids are found, and thresholding can be performed to eliminate any NPs that are outside size thresholds. The final step is an optional manual input to deselect any NP that aren't located correctly, and then a series of centroids have been obtained. While NP overlapping can cause difficulties for detection, the automated response provides quick and accurate centroid locations on a majority of NP in the image.

Furthermore, the NPs can be tracked between images in the tomography tilt series. Image stabilization is performed in case of translation during rotation, and then an appropriate particle tracking method is used to track individual NP through all of the images. This allows for the determination of NBD patterns from the same particle at different sample tilt angles.

Examples of three NBD patterns with pattern analysis performed are shown in Fig 2. To detect the Bragg reflection correctly, a morphological background subtraction is applied, followed by an automatic thresholding to separate the Bragg reflections from the background. Size thresholding is performed as well. Users can either allow the process to continue automatically, or can manually pick the locations of 
the $d_{1}$ and $d_{2}$ vectors in the NBD the pattern with mouse clicks. The software then determines the distances, angles and lengths of all three vectors that are used for measuring the unit cell distances of the particle.

The final step in the automated STEM / NBD data acquisition is an iterative optimization calculation to determine the unit cell parameters from the experimental NBD data. The three distance variables that make up the unit cell are iteratively modified and minimized against the measured values of the unit cell. The entire process, from a single image acquisition to diffraction pattern analysis, typically takes less than a minute for the data shown in Fig. 1 and 2.

An automated process for NBD / STEM nanoparticle location and 3D data collection significantly speeds up the characterization of samples containing discrete nanoparticles. The amount of data, while large, is smaller than in ptychographic methods or in atomic resolution electron tomography. The practical outcome is the identification of NPs and their structure in 3D when combined with electron tomography.

\section{References:}

[1] F. Paraguay-Delgado, M. Malac and G. Alonso-Nuñez, Materials Research Express 1 (2014), p. 24462-24467.

[2] M. Malac et al, Ultramicroscopy, 109 (2008), p. 14-21.

[3] M. Bergen et al, Microscopy and Microanalysis, 19 (2012), p. 1395.
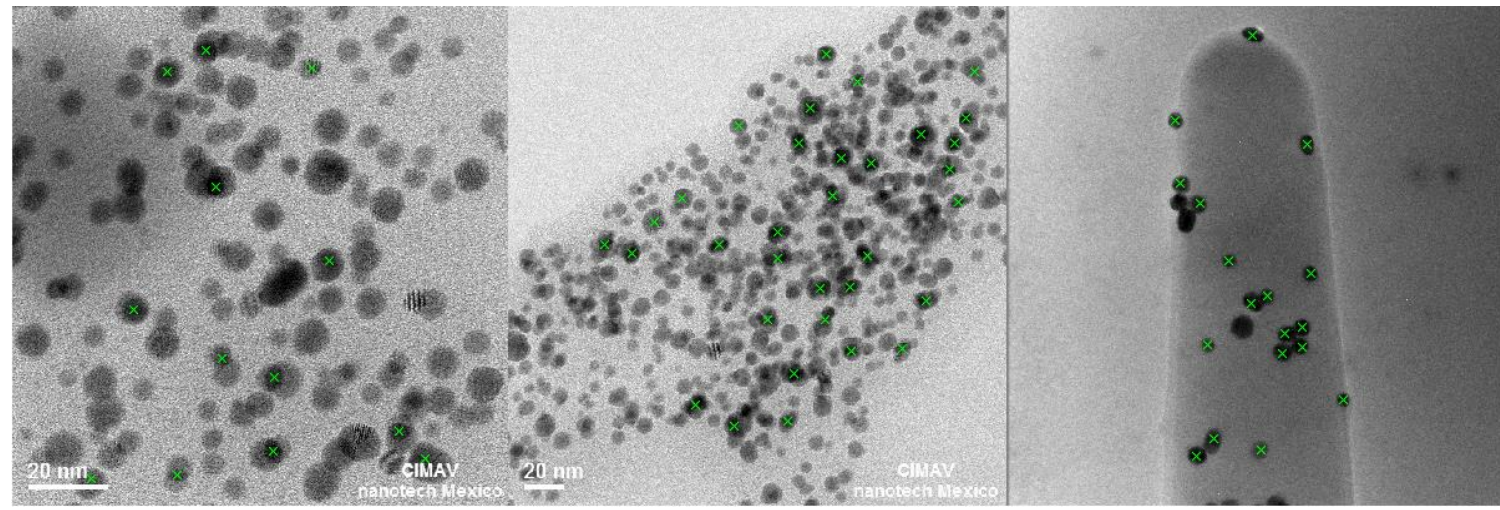

Figure 1. Three images of gold nanoparticles, with particle locations determined by the automation software algorithms and minimal user input.

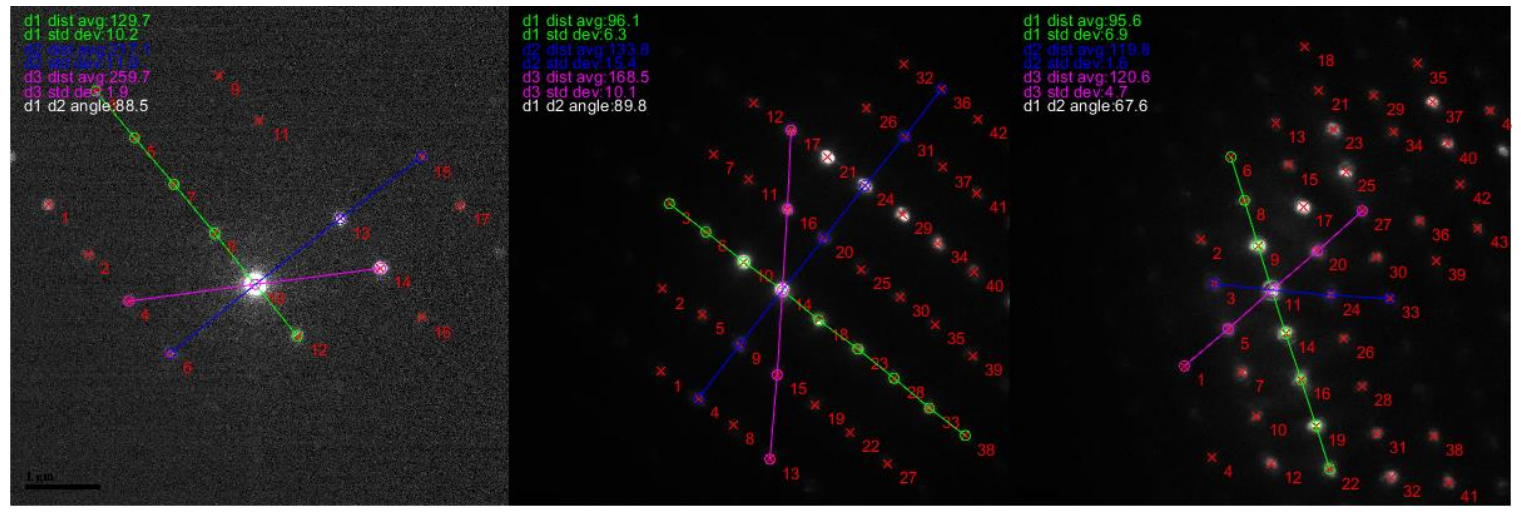

Figure 2. Diffraction patterns taken with NBD. Pattern detection and measurement are fully automatic and are calculated in a time frame on the order of seconds. 